\title{
Role of Single Dose Prophylactic Antibiotic in Elective Caesarean Section
}

\author{
Hasna Hena Pervin ', Rezaul Karim Kazal ${ }^{2}$, Kazi Farhana Begum ${ }^{1}$, Nilufar Islam ${ }^{1}$, Reefaat Rahman ${ }^{1}$ Rowshan \\ Ara $^{1}$ Farah Noor ${ }^{3}$ \\ ${ }^{1}$ Medical Officer, ${ }^{2}$ Associate Professor, ${ }^{3}$ Consultant, Department Of Obstetrics and Gynaecology, Bangabandhu Sheikh Mujib Medical University.
}

\begin{abstract}
:
Background: Maternal morbidity related to infection after caesarean section has been reported to be higher than that of vaginal delivery. The prevention of infection in patients undergoing caesarean section is a major challenge, particularly in hospitals, where there is frequent chance of cross infection due to overcrowding. Objective: The present study was undertaken to see the efficacy of single dose ceftriaxone as a prophylaxis in elective caesarean section to prevent postoperative infection. Methods: This cross sectional study was conducted on 100 patients who underwent elective caesarean section where single dose of Ceftriaxone was used as prophylaxis in the Department of Obstetrics \& Gynaecology, Bangabandhu Sheikh Mujib Medical University Hospital, between January to June 2010. The outcomes measures were postoperative febrile morbidity, wound infection and other infections (urinary tract infection, chest infection). Data were analysed using statistical package for social sciences (SPSS) version 11.5. Results: Over one-third (38\%) patients age group was between $18-25$ years, $56 \%$ inbetween $25-35$ years and $6 \%$ were over 35 years old. Nearly half $(46 \%)$ was anemic (haemoglobin $<11 \mathrm{~g} / \mathrm{dl}$ ) and $16 \%$ was obese $\left(B M I \geq 28 \mathrm{~kg} / \mathrm{m}^{2}\right)$. All the patients were operated by obstetricians of same level. Three percent patients developed wound infection, $5 \%$ developed other infections like UTI and chest infection and $7 \%$ had febrile illness. Among the several factors suspected to be associated with postoperative complications, preoperative anaemia, longer duration of operation ( $>60$ minutes) and prolonged hospital stay ( $>1$ week) were found to be significantly associated with postoperative complications. Conclusion: The present study suggests that single dose prophylactic antibiotic ceftriaxone given 1 hour before operation in patients with cesarean section deliveries reduces the chance of postoperative infection to a bare minimum.
\end{abstract}

Key words: Caesarean section, prophylactic antibiotics, postoperative complications.

[BSMMU J $2013 ; 6(1): 11-15]$

\section{Introduction:}

Caesarean section (CS) delivery is a surgical operation to deliver a baby through an incision in the uterus. Maternal morbidity related to infection after caesarean section has been reported to be 8 -fold higher than after vaginal delivery. Endometritis is the most common major complication occurring after caesarean section, with an incidence of $5-40 \%{ }^{2}$. A reduction of endometritis by two-thirds to three-quarters justifies the administration of prophylactic antibiotics to women undergoing caesarean section ${ }^{3,4}$. A systematic review in the Cochrane Library concluded that antibiotic prophylaxis in all cases of cesarean section significantly reduce the incidence of puerperal infection ${ }^{5}$.

Conventional antibiotic therapy carries a number of disad-

Address for Correspondence: Dr. Hasna Hena Pervin

Medical officer, Obs \& Gynae Dept, BSMMU.

E-mail address: hasnahenan@gmail.com, Mobile: 01811118105 vantages in terms of longer duration, potential side-effects and development of drug resistance incurred. To overcome these problems of conventional antibiotic, single dose antibiotic prophylaxis preoperatively at least one hour before operation was tested to control the infection ${ }^{6}$. The present study intended to see the efficacy of single dose ceftriaxone as a prophylaxis in elective caesarean section to prevent postoperative infection.

\section{Methods:}

This cross sectional clinical study was carried out in the Department of Obstetrics \& Gynaecology, Bangabandhu Sheikh Mujib Medical University Hospital, Dhaka over a period of six months between January to June 2006. A total of 100 patients who underwent elective caesarean section and consented in written to participate in the study were included as sample. However, patients with a history of hypersensitivity to cephalosporin group of drugs, history of having antibiotics within two weeks prior to the 
operation, any visible infection at any site or elevated temperature at the time of operation or history of any other chronic illness were excluded from the study.

A complete history of the participants was taken followed by physical examination to exclude any infection. A single dose of $1 \mathrm{gm}$ ceftriaxone was given 1 (one) hour before operation. The operations were performed by junior obstetricians (assistant registrar, indoor medical officer, honorary medical officer) as well as senior obstetricians (all obstetricians from consultant to professor). Following procedures were mandatory for all patients. Estimation of haemoglobin concentration on two occasions (preoperatively and on day 3 following operation). Pre-operative bladder catheterization done when needed. Lower segment caesarean section was done in every patient through a Pfannenstiel incision. During postoperative period $\left(1^{\text {st }}\right.$ to $5^{\text {th }}$ day $)$ information was collected including pulse rate, blood pressure, temperature, urinary complaints and status of wound (whether infected or not). Urine for routine microscopic examination was done in every patient on $3^{\text {rd }}$ post-operative day. Patients were considered obese if their BMI in the last trimester was $\geq 28$ $\mathrm{kg} / \mathrm{m}^{2,7}$ and anaemic if their hemoglobin concentration in peripheral blood was $11 \mathrm{gm} / \mathrm{dl}$ or less (According to WHO).

The outcomes were measured in terms of post-operative febrile morbidity (defined as oral temperature of $38.0^{\circ} \mathrm{C}$ on 2 occasions at least 4 hours apart, excluding the first 24 hours), Post-operative infection, which includes: wound infection (fever, cellulitis and exudates), endometritis (fever, uterine tenderness and abnormal lochia), peritonitis (elevated temperature, tachycardia, abdominal distension and pain with guarding and rigidity aggravated by moving and breathing with absence of bowel sound. Other febrile morbidity, e.g. urinary tract infection, chest infection were also looked for. Once febrile morbidity was identified, patients were examined to localize the potential site of infection (tonsils, breasts, chest, abdomen and pelvis). Urine analysis followed by urine culture and sensitivity testing was done if the result was suggestive of infection and a total white blood cell count was performed. Swab was taken from every infected wound for culture. If there was growth of microorganisms, they were isolated and drug sensitivity for each organism was tested and antibiotic was given accordingly. Data were analysed using statistical package for social sciences
(SPSS) version 11.5. The Chi-square $\left(\mathrm{x}^{2}\right)$ Probability test and Student's T-test was employed to analyse the data. Level of significance was set at 0.05 and $p$-value $<0.05$ was considered significant.

\section{Results:}

Demographic characteristics of the women selected for study showed that $38 \%$ study subject were in the age group $18-25$ years, $56 \%$ between $25-35$ years and the rest $6 \%$ over 35 years. Nearly half $(46 \%)$ were anemic (haemoglobin $<11 \mathrm{~g} / \mathrm{dl}$ ) and $16 \%$ was obese (BMI $\geq 28$ $\mathrm{kg} / \mathrm{m}^{2}$ ) (Table I). Fifty two percent of the patients were operated within $45-60$ minutes followed by $36 \%$ between $30-45$ minutes and $12 \%$ required more than 60 minutes (Table II). Of the 100 patients $42 \%$ were operated by junior obstetricians and $58 \%$ by senior obstetricians (Table III). Postoperatively $3 \%$ developed wound infection, $5 \%$ other infections like UTI and chest infection and $7 \%$ febrile morbidity (Table IV).

Among the several factors suspected to be associated with postoperative complications, preoperative anaemia, longer duration of operation ( $>60$ minutes) and prolonged hospital stay ( $>1$ week) demonstrated their significant presence in patients with postoperative complications. Significant number (11 out of 12) of patients who developed complications required more than 60 minutes to have their operations completed as opposed to minimum number ( 1 in 12) who did not develop the same ( $\mathrm{p}<$ $0.001)$. The mean hospital stay was also much higher in the former group than that in the later group. BMI and experience of surgeons were not observed as determinants of postoperative complications (Table $\mathrm{V}$ ).

Table-I

Baseline demographic and clinical characteristics $(n=100)$

\begin{tabular}{lcc}
\hline Age group & Frequency & Percentage \\
\hline $18-25$ & 38 & 38.0 \\
$25-35$ & 56 & 56.0 \\
$>35$ & 6 & 6.0 \\
BMI & & \\
$>28$ (Obese) & 16 & 16.0 \\
$<28$ (Non-obese) & 84 & 84.0 \\
Haemoglobin level & & \\
$<11$ gm/dl (anaemic) & 46 & 46.0 \\
$>11$ gm/dl (non-anaemic) & 54 & 54.0 \\
\hline
\end{tabular}


Table-II

Time required to perform operations $(n=100)$

\begin{tabular}{lcc}
\hline Duration (minutes) & Frequency & Percentage \\
\hline $30-45$ & 36 & 36.0 \\
$45-60$ & 52 & 52.0 \\
$>60$ & 12 & 12.0 \\
Total & 100 & 100.0 \\
\hline
\end{tabular}

Table-III

Level of the surgeons who performed operations $(n=100)$

\begin{tabular}{lcc}
\hline Surgeon & Frequency & Percentage \\
\hline Senior & 42 & 42.0 \\
Junior & 58 & 58.0 \\
Total & 100 & 100.0 \\
\hline
\end{tabular}

Table -IV

Distribution of patients by post-operative complications

\begin{tabular}{lcc} 
& $(n=100)$ & \\
\hline Complications & Frequency & Percentage \\
\hline Febrile morbidity & 7 & 7.0 \\
Wound infection & 3 & 3.0 \\
Other infections & & \\
(UTI, chest infection) & 5 & 5.0 \\
No complications & 85 & 85.0 \\
\hline
\end{tabular}

Table-V

Post-operative complications in patients with Associated factors

\begin{tabular}{|c|c|c|c|c|}
\hline \multirow[t]{2}{*}{ Variables } & \multicolumn{2}{|c|}{ Complications } & \multirow[t]{2}{*}{$\chi^{2}$} & \multirow[t]{2}{*}{$\mathrm{p}$-value } \\
\hline & Deve loped & Not developed & & \\
\hline \multicolumn{5}{|l|}{ BMI } \\
\hline Obese (16) & 3 & 13 & 0.006 & 1.0 \\
\hline Non-obese (84) & 12 & 72 & 0.006 & 0.827 \\
\hline \multicolumn{5}{|l|}{ Haemoglobin } \\
\hline Anaemic (46) & 12 & 34 & 8.213 & 0.914 \\
\hline Non-anaemic $(\mathrm{g} / \mathrm{dl})(54)$ & 3 & 51 & 8.213 & 0.003 \\
\hline \multicolumn{5}{|l|}{ Level of surgeon } \\
\hline Junior (42) & 6 & 36 & 0.029 & 1.0 \\
\hline Senior (58) & 9 & 49 & 0.029 & 0.781 \\
\hline \multicolumn{5}{|l|}{ Operative time } \\
\hline $30-60(88)$ & 4 & 84 & 62.864 & 0.000 \\
\hline$>60 \quad(12)$ & 11 & 1 & 62.864 & 0.001 \\
\hline
\end{tabular}




\section{Discussion:}

The high rate of infection following cesarean section (35-40\%) in different studies justified to consider prophylactic pre-operative antibiotic administration, although there is wide variation in type of antibiotics used, its time of administration and number of doses. The drug should have a wide spectrum of activity with reasonably good activity against pathogens likely to be present at the incision site. The dosage regimen should be designed to ensure adequate tissue levels at the time the operation begins or shortly thereafter. The duration of therapy should be short. The drug should be free of major side effects ${ }^{8}$. The present study carried out to test the efficacy of prophylactic antibiotic ( $1 \mathrm{~g}$ ceftriaxone given half an hour before operation) in reducing postoperative wound infection in elective caesarean section.

In the present study majority ( $85 \%)$ of the cases experienced uneventful recovery. Only $3 \%$ exhibited wound infection, $5 \%$ developed other infections (chest infection and UTI) and 7\% developed febrile morbidity of unknown etiology. In a similar study by Ahmed and Mirghani et al ${ }^{9}$ in their study found post-operative wound infection as 1.5 $\%$ and febrile morbidity as $7 \%$ with no patient suffering from endometritis or peritonitis bearing consistency with the findings of the present study. Agrawal et $\mathrm{al}^{10}$ also reported that patients in whom ceftriaxone was used had lower rate of wound infection $(4 \%)$. Woodfield et $\mathrm{al}^{11}$ found overall wound infection to be $8 \%$ and chest or urinary tract infection as $6 \%$ by using prophylactic ceftriaxone both in emergency and elective surgery. Incidences were somewhat higher than those found in the present study, on the other hand, findings of our study is supported by Smaill et al ${ }^{4}$ who had evidences from 17 trials of antibiotic prophylaxis for elective caesarean section confirming that prophylactic antibiotics reduce the risk of febrile morbidity or endometritis and wound infection. The rates of infection associated with elective caesarean section were lower than those of in emergency procedures.

Although the study demonstrated that prophylactic antibiotic (ceftriaxone) was able to prevent post-caesarean infection in majority of the cases, some (15\%) developed post operative infection. So risk factors made them vulnerable to acquire postoperative infections. Anaemic subjects are being more prone to develop postoperative infection than their non-anaemic counterpart. Longer duration of operation was also observed to be significantly associated with postoperative morbidity. These observations are consistent with those of Green and Sarubbi ${ }^{12}$, who found that patients with anemia and longer duration of operation, more frequently developed febrile complications after caesarean section. Nielson and Hokegard ${ }^{2}$ however, reported that anemia had no influence on complication rates in elective caesarean section.

This study did not find any significant association of post-operative complications with obesity and experience of the surgeon. This was consistent with the previous study by Nielson and Hokegard ${ }^{2}$. But obesity was revealed as a significant predictor for infection in emergency cases.

\section{Conclusion:}

We conclude with that prophylactic antibiotic ceftriaxone should be given 1 hour before operation in patients with cesarean section deliveries for reducing the chance of postoperative infection to a bare minimum ( 1 in 7 ). But protection against post-operative complications not only depends on preoperative antibiotic use but also on certain patient characteristics and operative time which must be taken into consideration to reduce the incidence of postoperative infection even further.

\section{References:}

1. Utupap K, Condon RE. Antibiotic prophylaxis for scheduled operative procedures. Infect Dis Clin N Am 1992; 6:613-25.

2. Nielson TF, Hokegard KH. Postoperative cesarean section morbidity: A prospective study. Am J obs gynecol 1983; 146:911-6.

3. Smaill F, Hofmeyr GJ. Antibiotic prophylaxis for caesarean section (Cochrane review). 2000.

4. Smaill F. Antibiotic prophylaxis and caesarean section. Brit J obs gynaecol 1992; 99:789-90.

5. Smaill F, Hofmeyr Gj. Antibiotic Prophylaxis for Cesarean (Cochrane Review). The Cochrane Library, Issue 1. Oxford: Update Software, 2002. 
6. Esposito $\mathrm{S}$. Is single dose antibiotic prophylaxis sufficient for any surgical procedure? N Engl J Med 1999; 11:556-64.

7. Dutta D C, Haematological disorders in pregnancy. Text book of obstetrics. $6^{\text {th }}$ Eddition, New central group book agency Pvt.

Limited, Kolkata - 700 009, India 2005; 262.

8. Alan H. DeCherney, Lauren Nathan. Antibiotic prophylaxis for cesarean section. Current Obstetric and Gynecologic Diagnosis and Treatment. 9th edition, The McGraw-Hill companies, Columbus, OH, USA. 2003: 547.

9. Ahmed ET, Mirghani OA, Gerais AS, Adam I. Ceftriaxone versus ampicillin / cloxacillin as antibiotic prophylaxis in elective cesarean section. East Mediterr Health J 2004; 10(3):227-88.

10. Agarwal M, Sah S, Agrawal M, Rastogi AN, Mukherji A, Sharma LB et al.Single dose cephalosporin (Ceftriaxone) as anti-microbial prophylaxis in surgical procedures, The Indian Practitioner 1997; 50(12): 1023-6.

11. Woodfield JC. A comparison of the prophylactic efficacy of ceftriaxone and cefotaxime in abdominal surgery. Am J Surg January $2002 ; 185: 45-9$.

12. Green S.L, and Sarubbi, FA Risk factors associated with post cesarean section febrile morbidity, Obstet. Gynecol 1977; 49:686-690 